SECTION 4. Computer science, computer engineering and automation.

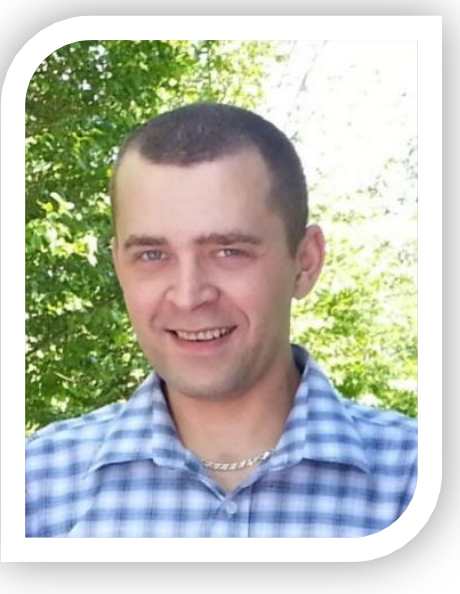

Rakhmatov Sukhrob Yuryevich

2 year student of the speciality "Computers and software"

Taraz State University named after M.Kh.

Dulati, Kazakhstan

\section{Shevtsov Alexandr Nikolayevich} candidate of technical Sciences, associate

Professor of the Department «Applied mathematics» Taraz State University named after M.Kh. Dulati, Kazakhstan

\title{
DEVELOPMENT OF AN ALGORITHM FOR REMOVING VIRUSES MOST COMMON IN TARSU
}

The article describes the process of creating anti-virus program to protect computers from USB intrusion, as well as some algorithms hacking viruses.

Keywords: virus, algorithm, Delphi.

\section{УДК 004.492}

\section{РАЗРАБОТКА АЛГОРИТМА УДАЛЕНИЯ ВИРУСОВ НАИБОЛЕЕ РАСПРОСТРАНЕННЫХ В ТАРГУ}

В статье рассматривается процесс создания антивирусной программы, для защиты компьютеров от USB вторжений, а также некоторые алгоритмы взлома вирусов.

Ключевые слова: вирус, алгоритм, Дельфи.

По данным исследований проводимых в первой половине 2012 года «Лаборатории Касперского» совместно с компанией O+K Research, до 70\% пользователей сети Интернет, так или иначе, сталкивались с 
деятельностью злоумышленников: страдали от вредоносного ПО, вирусов и др. [1]. При этом в 42\% случаев заражение компьютеров осуществляется именно вирусами Рис.1. (распространяющимися посредством USB хостов), $100 \%$ опрошенных нами пользователей заявили, что активно используют USB flash накопители для обмена данными между устройствами.
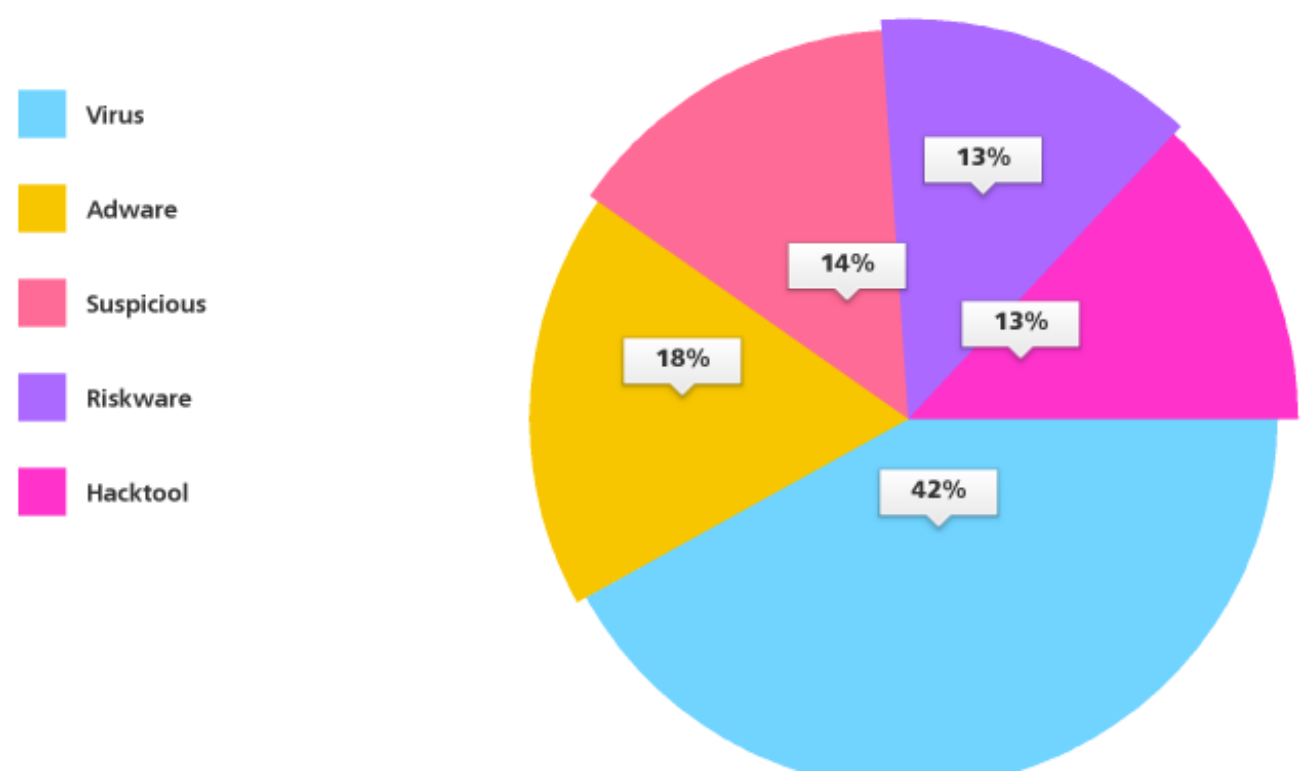

\section{Рисунок 1 - Различные классы угроз, выявленные на компьютерах пользователей в сентябра 2012 года. [2]}

В проведенном нами исследовании в течении последнего месяца в компьютерных аудиториях ТарГУ и на кафедрах, наиболее часто встречались следующие вирусы:

recycler, velike, mco.sys, ntr.svc,mizelje.exe, fswagz.exe, cbzvl.exe, nsvb.exe, ciadmin.htm, ciquery.htm, malicrni, marijin, nijetebi, dosebe.exe, ziaipe.exe, Sexy.exe, Porn.exe, Passwords.exe, Secret.exe, ziaipe.exe, DIJANA, bembara.exe, evonocas, nisamtebe.exe, ...exe, DrWebQuarantine.exe, ckdiip.exe, $u \partial p$.

Большая часть из них относится к категории троянов и червей, Рис.2, и имеет общий для всех, характер действий и методы заражения. Исследования методов заражения и разработка способов противодействия и удаления вирусов проводились на отдельных компьютерах, и в компьютерных аудиториях. 


\section{Найденные вирусы \\ dazid.exe \\ faomip.exe \\ geazio.exe \\ guemej.exe \\ jzsun.exe \\ liueh.exe \\ mnfox.exe \\ puenoe.exe \\ quula.exe \\ rooasay.exe \\ Рисунок 2 - Вирусы из категории троянов и червей распространяющиеся посредством USB накопителей}

Антивирусные программы Esetnod 32, Kaspersky 6 версии, Dr. Web и др. используемые в компьютерных аудиториях университета довольно часто не имеют последних обновлений антивирусных баз. А даже и при наличии таковых, имеют выявленные в процессе исследования недостатки, не могли обнаружить активные вирусы, и не могли обеспечить надлежащую защиту компьютера. Так при работающем антивирусе EsetNod 32 (4 версия) в аудитории 2.4.208 были обнаружены 2 вируса: qiiat.exe и waujoo.exe которые находились в активном режиме и при этом Nod 32 их не обнаруживал. После нашего вмешательства и изменении настроек оперыционной системы Nod 32 получил больше привилегий и после указания на местоположение вирусов смог их удалить.

По результатам исследования были выделены следующие общие черты вирусов относящихся к «Червям» и «Троянам»: заражая компьютер они дислоцировались в определенных местах, меняли настройки ОС, блокировали активные программы и антиувирусы, меняли реестр, добавляли «себя» в автозагрузку, а в отдельных случаях меняли файловую структуру данных. Тем самым представляя непосредственную опасность . Нами были рассмотрены три алгоритма удаления вирусов, с зараженного компьютера: KillTask, KillProgram, WipeFile. Bсе они были реализованы в Delphi и опробаваны как на неактивных вирусах, так и на активных.

В случае неактивной стадии вируса, когда он пассивен и не пытается вмешиваться в работу операционной системы, реестра и файловой структуры, все три алгоритма оказались полностью работоспособными Рис.3.

В случае активного противодействия вируса антивирусной программе, и действиям пользователя - все три алгоритма показали свою несостоятельность Рис. 4.

В связи с этим нами был разработан новый алгоритм удаления Restart который позволил избежать подобной ошибки и гарантированно удалять активные вирусы Рис.5. 

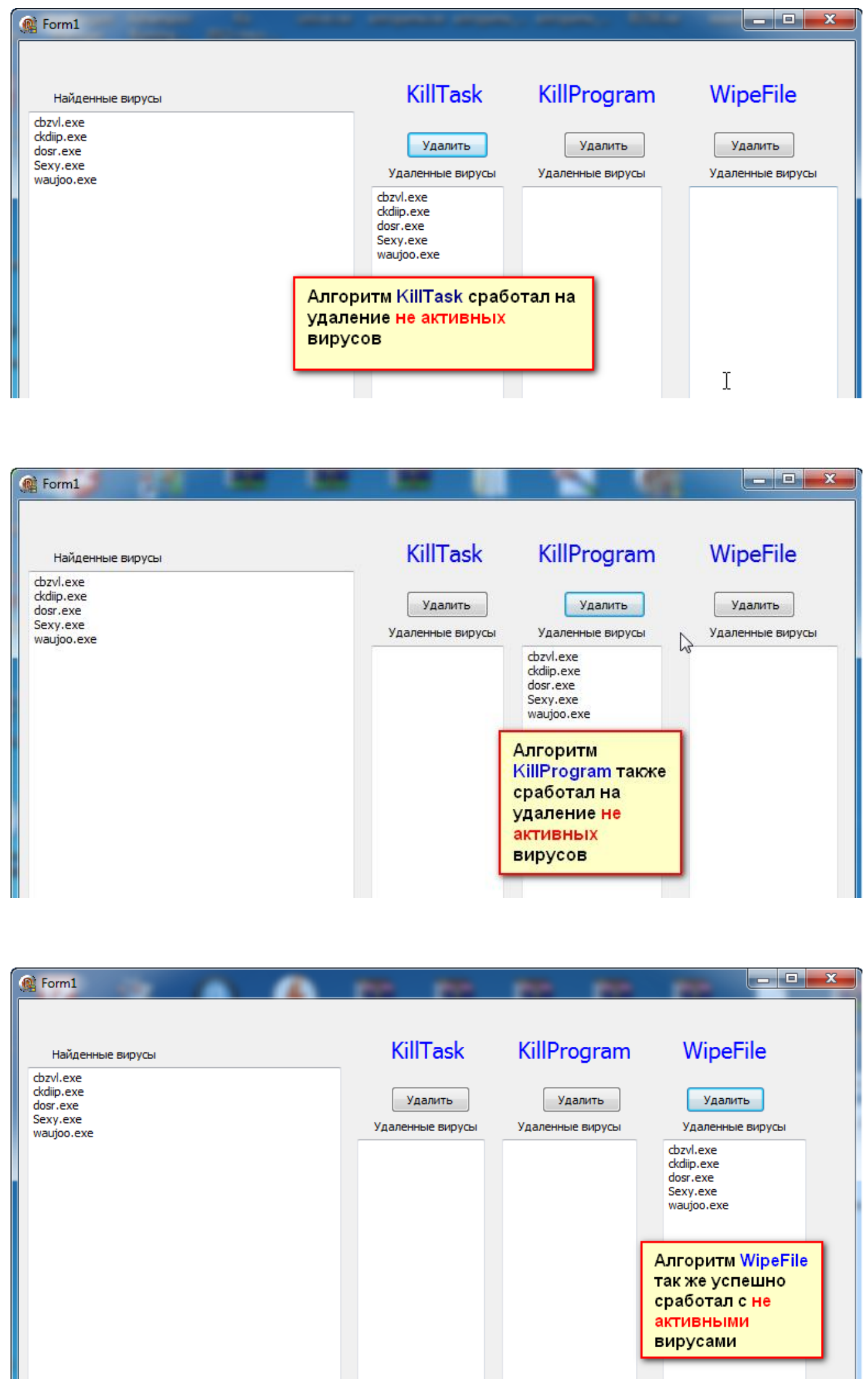

Рисунок 3 - Алгоритмы удаления не активных вирусов. 


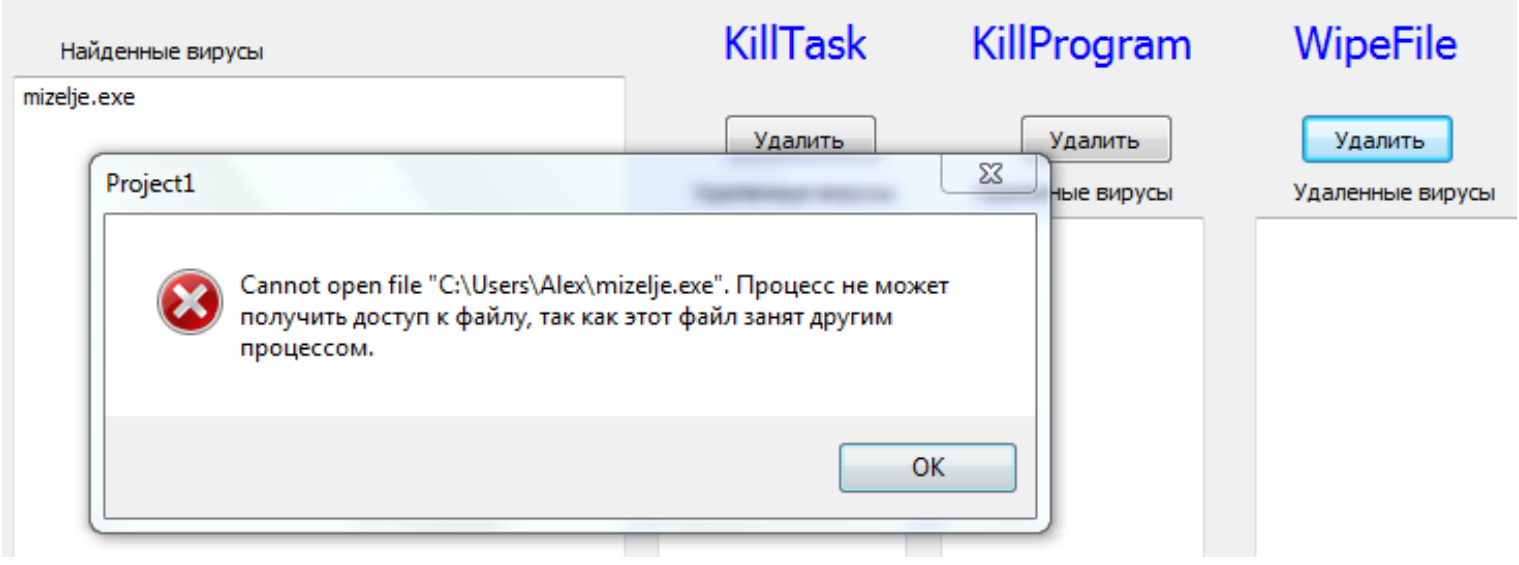

Рисунок 4 - Ошибка в процесе работы алгоритмов.

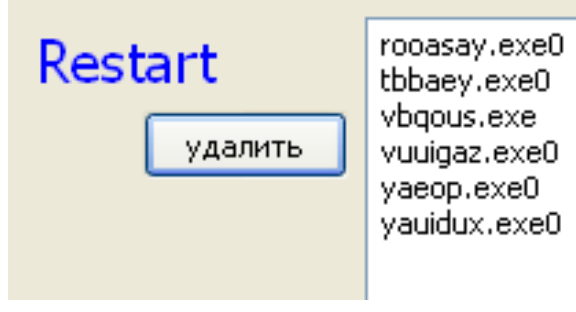

\section{Рисунок 5 - Алгоритм Restart.}

Алгоритм был разработан на Delphi и объединил в себе процесс поиска вирусов, удаление вируса и очистку реестра:

uses

...... FileCtrl, ShlObj,Thelp32, Registry;

var

......s,sn:string; i:integer;

function restart(s0:string):boolean;

var a:TRegistry;

begin

a:=TRegistry.Create;

a.RootKey := HKEY_LOCAL_MACHINE;

a.OpenKey('ISOFTWARE\Microsoft \WindowslCurrentVersion\RunOnce', false);

$$
\text { if } \mathrm{s} 0<>" \text { then }
$$

a.WriteString('del'+s0,'command.com /c del "'+s+'l'+s0+'"'); if $\mathrm{s} 0<>"$ then 
a.WriteString('del0'+s0,'cmd /c del "'+s+'l'+s0+'"');

a.CloseKey;

a.Free;

if $\mathrm{s} 0<>"$ then result:=true;

end;

procedure TForm1.Button4Click(Sender: TObject);

begin

Load;

\{Поиск вирусов и загрузка списка найденных в Mето1\}

for $\mathrm{i}:=0$ to memo1.Lines.Count do

begin

sn:=memo1.Lines.Strings[i];

if $s n<>"$ then

if restart(sn) then memo5.Lines.Add(sn);

end;

for $i:=0$ to form1.memo1.Lines.Count do

begin

sn:=form1.memo1.Lines.Strings[i];

FileSetHidden(s+'l'+sn,false);

end;

if MessageDlg('Перезагрузитьсейчаc?',mtCustom,[mbOk,mbCancel], 0)= mrOk then MyExitWindows(EWX_REBOOT or EWX_FORCE); end;

\begin{tabular}{|c|c|c|c|c|c|c|}
\hline \multicolumn{2}{|c|}{ USB US B - 2012} & \multicolumn{3}{|c|}{ версия - 3.2} & & $-\square \times$ \\
\hline \multicolumn{7}{|c|}{ Файл Помощь } \\
\hline \multirow{2}{*}{\multicolumn{2}{|c|}{ Диск F: }} & \multicolumn{3}{|c|}{ Проверка } & & \\
\hline & & \multirow{2}{*}{\multicolumn{3}{|c|}{ Активная защита ! }} & & \\
\hline 囯 & $:[]$ & & & & & \\
\hline \multicolumn{2}{|c|}{ Статистика } & База & Агрессивная защита & \multicolumn{2}{|c|}{ Лечение Флешки } & \\
\hline & Имя файла & & Размер файла & Действие & Путь & Время \\
\hline 1. & autorun.inf & & $0 \kappa 6$ & Удален & F:lautorun.inf & $15: 37: 21$ \\
\hline 2. & Irjuiz.exe & & $180 \kappa 6$ & Удален & F:'illijuiz,exe & $15: 37: 25$ \\
\hline 3. & Irjuizx.exe & & $180 \kappa 6$ & Удален & F:lilijuizx.exe & $15: 37: 25$ \\
\hline 4. & WPaapouc.exe & & $133 \kappa 6$ & Удален & F:IRECYCLER!' & $3: 15: 37: 27$ \\
\hline
\end{tabular}

\section{Рисунок 6 - Антивирус «USB - 2012».}

Полученный алгоритм предполагается внедрить в антивирусную программу «USB - 2012», разработанную на кафедре «Прикладная 
математика» в ТарГУ, обеспечивающую активную защиту от вторжений вирусов через USB, расширив тем самым ее функциональность Рис.6.

\section{ЛИТЕРАТУРА}

1. «Лаборатория Касперского» на Неделе Российского Интернета: киберугрозы в Рунете и как с ними бороться. - Russian Internet Week, RIW 2012), Москва 17-19 октября 2012г. [Электронный ресурc]. URL: http://www.kaspersky.ru/news?id=207733866 $\quad$ (дата обращения: 27.04.2013).

2. Обзор вирусной активности в сентябре 2012 года: новое семейство опасного троянца и программы-шпионы для мобильных устройств. 1 октября 2012г. [Электронный ресурc]. URL:

http://news.drweb.com/?i=2827\&c=5\&lng=ru\&p=1 (дата обращения: 27.04.2013). 